\title{
Magnetoelastic effects in low-dimensional magnetic systems
}

\author{
A. A. Zvyagin ${ }^{1,2}$ and G. A. Zvyagina ${ }^{2}$ \\ ${ }^{1}$ Max Planck Institut fur Physik Komplexer Systeme, Nöthnitzer Str, 38, D-01187, Dresden, Germany \\ ${ }^{2}$ B. Verkin Institute for Low Temperature Physics and Engineering of the National Academy of Sciences of Ukraine \\ 47 Lenin Ave., Kharkov 61164, Ukraine
}

Received July 25, 2000

\begin{abstract}
We consider a new realization of magnetoelastic interactions in low-dimensional magnetic systems. We show that low-dimensional spin systems are unstable with respect to the spontaneous appearance of alternating distortions of the positions of the three-dimensional nonmagnetic atoms (ligands), that surround the magnetic ions. Those distortions are supplemented by the spontaneous onset of alternating effective $g$ factors of the magnetic ions in the phase with short-range interactions. We discuss the possibility of observing the effect in an uniform external magnetic field, which in the situation considered produces both magnetization and staggered magnetization of the magnetic subsystem. The connection of the proposed theory with recent experiments on effectively low-dimensional magnetic systems (organic spin chains, heavy-fermion compounds, rare-earth molybdates) is discussed.
\end{abstract}

PACS: 75.10.Jm, 71.70.Fk, 71.70.Ej

Interest in electron systems with substantial coupling between the charge, spin, and orbital degrees of freedom of the electronic and elastic subsystems of a crystal has grown considerably during the last decade. The prime examples of the manifestation of such cooperative effects are the phenomena of collosal magnetoresistance of manganites, non-Fermiliquid behavior of some heavy-fermion compounds, spin-Peierls and charge ordering behaviors in inorganic systems and unconventional superconductivity [1]. The Jahn-Teller effect [2] is probably the oldest known manifestation of such a coupling. Here the degeneracy of the orbital states of a molecule is removed by the deformation of the latter. The cooperative Jahn-Teller effect reveals itself in a structural phase transition. It has been observed in a number of compounds [3]. In the spin-Peierls transition also the degeneracy of the electronic (spin, not orbital) subsystem of a onedimensional $(1 D)$ spin chain is removed due to the coupling with a longitudinal phonon of the $3 D$ crystal lattice. A spin gap is opened for the lowlying spin excitation. On the other hand, the corresponding phonon mode possesses softening (Kohn anomaly). Some of magnetic compounds with the essential coupling between spin, orbital, and elastic subsystems manifest paramagnetic spin behavior with two essentially inequivalent magnetic centers at low temperatures (which are higher, though, than the temperature of the phase transition to magnetically ordered $3 D$ state). Often the latter has not been observed [4-9]. For higher temperatures the inequivalence between the two magnetic centers smears out. The presence of inequivalent magnetic centers in low-dimensional quantum spin systems is usually connected with slightly different local surrounding of two types of magnetic ions and involves either staggered $g$ factors of the magnetic ions [4] (another case pertains to two anisotropic $g$ tensors canted with respect to the principal axis [8] or the Dzyaloshinskii-Moriya coupling in crystals without mirror magnetic symmetry [10]. Low-lying spin excitations are gapless for these low-dimensional systems in the absence of an external magnetic field. However,in an external field the lowtemperature specific heat of some of those systems reveals the emergence of a spin gap induced by an external magnetic field $[8,11,12]$. To explain that gapped behavior a $1 D$ spin subsystem with staggered effective $g$ factors has been stadied [5,13,14]. The low-temperature, low-field electron spin resonance (ESR) [4,6-9] exhibited two inequivalent magnetic centers. On the other hand, the higher-field ESR treatment reported only 
a single magnetic center [15]. Optical (Raman and infrared) measurements [16] have revealed the tendency for softening of some lattice modes. Ultrasonic measurements also revealed anomalous behaviors of the sound velocities for some phonon modes in such crystals $[17,18]$.

In this work we propose a mechanism for explanation of the observed anomalous behavior of the spin, orbital, and elastic subsystems of that group of magnetic low-dimensional systems. Namely, we point out that the dimerization of the effective $g$ factors in a quantum antiferromagnetic spin chain can be caused by the coupling of the spin and orbital subsystems to the elastic subsystem, followed by a distortion of the local surrounding of the magnetic ions. In paramagnets the value of the $g$ factor differs from 2 . The difference stems from the effect of the crystalline electric field of nonmagnetic ligands due to the presence of a spin-orbit interaction. The essence of our study is following. Let the configuration of ligands, which surround two neighboring magnetic ions along the chain direction, possesses small shifts (distortions) of opposite signs (antiferrodistortions [8]). Such distortions will immediately produce a change of the crystalline electric fields of the ligands. Hence, the orbital moment of the magnetic ions will be alternately affected. Then the spin-orbit interaction yields, first, two different values of the effective $g$ factors of the magnetic ions, and second, a staggered Dzyaloshinskii-Moriya interaction (because of the odd magnetic symmetry with respect to the principal axis). The effect of the latter can be also transferred to the effective staggered $g$ factor by means of an alternating rotation of spins. Hence, the staggered distortion of the nonmagnetic surroundings (ligands) of the magnetic ions produces two inequivalent magnetic centers for the spin subsystem. The elastic subsystem loses energy due to the alternating distortions of ligands, while the electron subsystem realizes an energy gain. The steady-state configuration of the total crystal is determined by the competition between those two processes. From the magnetic standpoint that effect is collective because the spins interact with each other. On the other hand, the inequivalence of magnetic centers can be seen only in nonzero magnetic field, so the effect is field-induced.

To fix the stage, consider the low-temperature behavior of a quasi-low-dimensional magnetic crystal. The spin-spin coupling along one direction of this crystal is larger than the interactions along the other crystallographic directions. Suppose this quasi- $1 D$ spin subsystem can be described by a
Hamiltonian of a gapless spin-1/2 antiferromagnetic (AF) chain. The fact that the low-lying excitation of the $1 D \mathrm{AF}$ spin chain is gapless implies that the system is critical, i.e., the ground-state correlation functions decay in a power-law manner. Suppose also that for the reasons explained above, some components of the effective $g$ factors of the spins are alternating, i.e., $g_{1,2}=g(1 \pm \delta)$, where $\delta<<1$ is proportional to the small alternating distortions of the local environment. The parameter $\delta$ is not fixed, but has to be determined from the conditions of the steady state. It is equivalent to the mean field approximation for phonons. It is justified for the $3 D$ elastic subsystem. The $1 D$ spin-spin interactions will be taken into account nonperturbatively. In the case of alternating Dzyaloshinskii-Moriya interactions present in the spin chain the alternating effective $g$ factors are $g_{1,2}=g \times$ $\times \cos \vartheta(1 \pm \tan \vartheta)$, where the angle $\vartheta$ originates from, e.g., the alternating components of the crystalline electric field of the ligands [14]. For rareearth molybdates, where the $g$ tensors for two centers are canted with respect to the main axis, this means that $g_{1,2}=g \cos \alpha \cos \theta(1 \pm \tan \alpha \tan \theta)$ [8], where $\alpha$ and $\theta$ are the angles determining the canting of the $g$ tensors and field direction, respectively. The application of a field parallel to the crystallographic axes does not change the distortion. For rare-earth ions (with a Kramers- or nonKramers-doublet crystalline field ground state) for sufficiently low temperatures $T$ compared with the crystalline-field splitting $D$, one can use the two lowest levels of the ion as an effective spin-1/2 with magnetically anisotropic behavior. It turns out that no change of the $g$ factors can be produced by distortions of ligands for the ground-state doublets. They do not have orbital degrees of freedom and are therefore unaffected by distortions. However, the excited crystalline electric field states are effectively included. This is why a distortion of the ligands can produce a change of the $g$ factors, through the off-diagonal matrix elements of the relevant electric-multipole operators. This means that the conditions for the applicability of our effective spin- $1 / 2$ description for rare-earth compounds are: (i) $T<<D$, i.e., there is no thermal population of the crystalline electric field-excited levels; (ii) off-diagonal matrix elements (between the ground-state doublet and excited levels) of the Zeeman term must be small compared with $D$; (iii) the matrix elements between the ground-state doublet and crystalline electric field-exited levels of the operator describing the distortion must not be too small compared to $D$ (otherwise distortions 
would produce a negligible change of the groundstate $g$ factors); (iv) distortions of the ligands must not affect the exchange constant $J$; see below (with $J<<D$ ).

The Hamiltonian can be written as

$$
\begin{aligned}
\mathcal{H}_{\mathrm{sp}} & =J \sum_{j}\left(\mathbf{S}_{j} \mathbf{S}_{j+1}+(\Delta-1) S_{j}^{z} S_{j+1}^{z}\right)- \\
& -\mu_{B} H \sum_{j}\left(g_{1} S_{2 j}^{z}+g_{2} S_{2 j+1}^{z}\right),
\end{aligned}
$$

where $0 \leq \Delta \leq 1$ is the parameter of the «easyplane» magnetic anisotropy, $H$ is an external magnetic field, and $\mu_{B}$ is the Bohr magneton. It is easy to show that because of nonzero $\delta$ the application of an external field to this system yields two effects: homogeneous magnetization $\left(\mathrm{m}^{z}\right)$ and staggered magnetization. The former changes the ground-state filling of the Dirac sea ( $H$ plays the role of the Fermi energy). For large enough values of the field $H$ the spin subsystem possesses trimerization (for the Dirac sea of spinons being one-third filled), quadrimerization (for one-fourth filling), etc. For those values of the field $2 n k_{F}=\pi$, with $n$ being an integer and $2 k_{F}=\pi\left(1+2 m^{z}\right)$, i.e., we expect the series of transformations towards an inhomogeneous (incommensurate) magnetic structure due to the umklapp processes rather than the dimerization [19]. We restrict ourselves to small enough values of $H$ here. The staggered magnetization is the relevant perturbation from the renormalization group (RG) viewpoint. It produces the gap for low-lying magnetic excitations of the system (spinons). We can calculate the response of our (critical) spin chain to the relevant perturbation (staggered field). We perform such a calculation in a RG framework. Our study shows that the exponents are non-integer in general, in contrast to simple perturbation or mean-field theories [8,16], which of course are not legitimate for the low-dimensional quantum spin systems. An application of scaling relations provides a simple tool to understand some essential aspects of the behavior of a critical chain under a relevant perturbation. Recall that response of the free energy $f_{\mathrm{cl}}$ and the correlation function $\xi_{\mathrm{cl}}$ of a classical critical $d$-dimensional system perturbed by a relevant operator $\delta \mathcal{H}^{\prime}$ with RG eigenvalue $v^{-1}>0$ is

$$
\Delta f_{\mathrm{cl}} \propto \delta^{d v}, \quad \xi_{\mathrm{cl}} \propto \delta^{-v} .
$$

A quantum critical $d$-dimensional system (which in our case is the spin $1 D$ subsystem of the crystal) formally behaves in the scaling regime equivalently to a $(d+z)$-dimensional classical system, where $z$ is the dynamical critical exponent. Hence, the groundstate energy and the gap of the low-lying spin excitations of the $d$-dimensional quantum critical system are formally proportional to the free energy and to the inverse correlation function of the $(d+z)$-dimensional classical critical system, respectively. The RG eigenvalue $v^{-1}$ is related to the scaling dimension $x$ of the particular operator by $x+v^{-1}=d+z$. For the (conformally invariant) $\mathrm{AF}$ spin chain we have $d=z=1$, i.e., $\mathrm{v}=(2-x)^{-1}$. Hence the renormalization of the ground-state energy per site of the quantum critical chain and the low-lying spin excitation gap (which is equal to zero at the unperturbed point) to the staggered magnetic field are

$$
\Delta E_{q} \propto-\left(g \delta \mu_{B} H\right)^{2 /\left(2-x_{e}\right)}, \quad \Delta \propto\left(g \delta \mu_{B} H\right)^{1 /\left(2-x_{e}\right)},
$$

respectively, where $x_{e}$ is the minimal scaling exponent for energy - energy correlations. Here we have ignored logarithmic corrections. They are present due to the marginal operators in the RG sense and essential for the $S U(2)$-symmetric case. To find the scaling dimension for our critical spin chain we use the results of the conformal field theory (CFT). According to the CFT approach [20] the asymptotics of the correlation functions of primary fields in the ground state are known to be $\left\langle\phi_{\Delta^{ \pm}}(r, t) \phi_{\Delta^{ \pm}}(0,0)\right\rangle=\exp \left(2 i D P_{F} r\right)\left(r-i v_{F} t\right)^{-2 \Delta^{+} \times}$ $\times\left(r+i v_{F} t\right)^{-2 \Delta^{-}}$, where $v_{F}$ and $P_{F}$ are the Fermi velocity and the Fermi momentum, respectively [a (half-)integer $D$ measures the momentum of the primary field in units of the Fermi momentum]. The scaling dimension and spin for each primary field are determined by $x_{\phi}=\Delta^{+}+\Delta^{-}$and $s_{\phi}=\Delta^{+}-\Delta^{-}$. The parameters $\Delta^{ \pm}$can be calculated according to the finite-size analysis of the low-energy physics of the critical spin chain. Combining all the effects, we can write for the ground-state energy of the spin subsystem with nonzero $\delta$

$$
E_{\mathrm{sp}}=-v_{F}\left(\frac{g \delta \mu_{B} H}{v_{F}}\right)^{2 /\left(2-x_{e}\right)}
$$

where for our $\mathrm{AF}$ spin chain $v_{F}=\pi J / 2 \times$ $\times \sqrt{1-\Delta^{2}} \cos ^{-1} \Delta$ is the Fermi velocity of spinons for $H=0$. We point out that the scaling approach is only valid in the vicinity of the critical point, i.e., in principle the values of the magnetic field and $\delta$ are small. Note that the Fermi velocity monotonically decreases with the growth of $H$ and becomes zero at the spin-saturation point (for 
$\left.H \geq H_{c} \sim J \Delta / 4 g \mu_{B}\right)$. The exponent is equal to $x_{e}=\pi / 2\left(\pi-\cos ^{-1} \Delta\right)$ for $H=0$ (it increases monotonically with the increase of $H$ and becomes 1 at $H=H_{c}$ ) [21]. Obviously $E_{\mathrm{sp}}<0$, and hence one has an energy gain due to nonzero $\delta$. On the other hand, in the lowest order in $\delta$ the elastic subsystem loses an energy proportional to $C \delta^{2} / 2$, where $C$ is the elastic constant. Whether the ground-state steady-state configuration corresponds to zero or nonzero $\delta$ depends on the scaling exponent $x_{e}$. For $x_{e}<1$ the ground-state steady-state configuration of the spin and elastic subsystems corresponds to nonzero $\delta$, and hence, to two inequivalent spin centers (with two different $g$ factors) and to the nonzero alternating distortions (antiferrodistortions) of the local environment of the magnetic ions (ligands). (Here we take into account that $|\delta|<1$.) The steady-state $\delta$ is equal to

$$
\delta_{0}=\left(\frac{v_{F}}{C}\right)^{\frac{2-x_{e}}{2\left(1-x_{e}\right)}}\left(\frac{g \mu_{B} H}{v_{F}}\right)^{\frac{1}{1-x_{e}}} .
$$

Clearly $\delta_{0}=0$ for $H=0$ and for $H \geq H_{c}$. This is the main result of our report. For high temperatures (much higher than $J$ ), the steady-state $\delta$ is zero, naturally. Hence, there has to be a phase transition between the low-temperature phase with nonzero staggered magnetization, gapped low-lying spin excitations, and nonzero alternating distortions of the ligands, surrounding the magnetic ions, and the high-temperature phase in which the staggered magnetization is zero, low-lying spin excitations are gapless and there are no distortions of the ligands. In fact we have considered the effect under a tacit assumption that the initial frequencies $\omega_{0}$ of the phonons which are coupled to the spin chain are small $\omega_{0}<J$ (adiabatic approximation). It is in principle possible to calculate the effect more precisely for any $\omega_{0}$ [22,23]: there the conditions for the steady-state configuration of the total system correspond to the cross section of the respective phonon modes $\omega(q)\left(\right.$ at $\left.q=2 k_{F}\right)$ connected to the alternating distortions (antiferrodistortions) of ligands with the magnetic modes [22,23].

Let us consider the interesting (RG-marginal with $x_{e}=1$ ) limiting case, namely $\Delta=0$. That case corresponds to the XX spin-1/2 chain with alternating $g$ factors. The Hamiltonian of the latter can be exactly mapped by means of the nonlocal Jordan-Wigner transformation on the Hamiltonian of the lattice noninteracting fermions [24]. The ground-state energy of the spin subsystem and the elastic subsystem can be written in the form

$$
\begin{gathered}
E_{g s}=\frac{C \delta^{2}}{2}-g \mu_{B} H\left[1-\frac{\lambda c}{\pi}\right]- \\
\lambda_{c} \\
-\frac{1}{\pi} \int_{0} d \lambda \sqrt{\left(\delta g \mu_{B} H\right)^{2}+[J \cos (\lambda / 2)]^{2}},
\end{gathered}
$$

where $\lambda_{c}=\cos ^{-1}\left[2\left(1-\delta^{2}\right)\left(g \mu_{B} H / J\right)^{2}-1\right]$. Minimizing the ground-state energy with respect to $\delta$ we obtain two possible solutions. The first one, $\delta=0$, corresponds to zero distortions. As to the second one, it satisfies the equation

$$
\frac{\pi C \sqrt{J^{2}+\left(\delta g \mu_{B} H\right)^{2}}}{\left(2 g \mu_{B} H\right)^{2}}=F\left(\frac{\lambda_{c}}{2}, k\right),
$$

where $F\left(\lambda_{c} / 2, k\right)$ is the incomplete elliptic integral of the first kind with $k^{-1}=\sqrt{1+\left(\delta g \mu_{B} H / J\right)^{2}}$. Notice that for $H \geq H_{c}=J / g \mu_{B} \sqrt{1-\delta^{2}}$ one has $\lambda_{c}=\pi$, and the integral is zero (hence for $H \geq H_{c}$ there is only one $\delta_{0}=0$ solution in the ground state, as should be the case). We emphasize again that we are studying the effect of sufficiently weak magnetic fields, for which, on the one hand, the scaling approach is valid, and, on the other hand, we restrict consideration to the dimerization only, i.e., the field is not so strong as to produce a one-third filling of the Dirac sea, etc., or a series of transformations into inhomogeneous magnetic phases. Equation (7) can be re-written in the form

$$
\sqrt{1-\left(1-\delta^{2}\right)\left(g \mu_{B} H / J\right)^{2}}=\operatorname{sn}(u, k),
$$

where $\quad u=\pi C \sqrt{J^{2}+\left(\delta g \mu_{B} H\right)^{2}} /\left(2 g \mu_{B} H\right)^{2} \quad$ and sn $(u, k)$ is the Jacobi elliptic sine function. This equation can be solved numerically. For $k \sim 1$ (which corresponds to small $\delta$ ), the asymptotic behavior of the elliptic function is known to be $\operatorname{sn}(u, k) \approx \tanh u+\left(1-k^{2}\right)(\sinh u \cosh u-u) / 4 \cosh ^{2} u$. (For large $k$ one has $\operatorname{sn}(u, k) \approx \sin u-k^{2} \times$ $\times \cos u(u-\sin u \cos u) / 4$.) It is possible to write an analytical asymptotical expression for the ground-state steady-state nonzero $\delta_{0}$ as

$$
\delta_{0} \approx\left(2 J / g \mu_{B} H\right) \times
$$

$$
\times\left(\frac{A \tanh u_{0}-A^{2}}{2-A \tanh 2 u_{0}-\left(A u_{0} / \cosh ^{2} u_{0}\right)}\right)^{1 / 2},
$$

where $A=\sqrt{1-\left(g \mu_{B} H / J\right)^{2}}$ and $u_{0}=\pi C J \times$ $\times\left(2 g \mu_{B} H\right)^{-2}$. It is clear that nonzero $\delta_{0}$ can appear 
only for $\tanh u>A$. For nonzero temperatures we calculate the free energy of our system (assuming that the Debye energy of the phonons is large, and, therefore the elastic subsystem effectively remains in the ground state)

$$
F=\frac{C \delta^{2}}{2}-\frac{T}{\pi} \sum_{ \pm} \int_{0}^{\pi} d \lambda \ln \left[2 \cosh \left(\frac{\varepsilon_{ \pm}(\lambda)}{2 T}\right)\right]
$$

where

$\varepsilon_{+}(\lambda)=g \mu_{B} H \pm \sqrt{\left(\delta g \mu_{B} H\right)^{2}+[J \cos (\lambda / 2)]^{2}}$. Then the minimization of the free energy with respect to $\delta$ yields

$$
C \delta=\frac{1}{2 \pi} \sum_{ \pm} \int_{0}^{\pi} d \lambda \tanh \frac{\varepsilon_{ \pm}(\lambda)}{2 T} \frac{\partial \varepsilon_{ \pm}(\lambda)}{\partial \delta}
$$

For $T>>J$ we can replace the hyperbolic tangent by its argument, and one clearly sees that there exists only one solution to Eq. (11): $\delta_{0}=0$. The numerical analysis shows that $\delta_{0} \neq 0$ exists at low temperatures. The critical temperature $T_{c}$ of the transition between the low-temperature (effectively two-center) phase and the uniform high-temperature phase is obtained by setting $\delta_{0}=0$ for the second order phase transition, or the free energies of the phases with $\delta_{0}=0$ and $\delta_{0} \neq 0$ for the first-order one. The critical temperature can be estimated (with the main input to the integral given by the divergent denominator, i.e., at the van Hove singularities) as

$$
T_{c} \sim\left(J-g \mu_{B} H\right) / \ln \left(\pi C J / 2 g^{2} \mu_{B}^{2} H^{2}\right) .
$$

The analysis shows that $\delta_{0}=0$ pertains to the minimum of the free energy for $T>T_{c}$ and to the maximum for $T<T_{c}$. Hence the phase transition is of the second order. We again emphasize that the phase transformations studied here are magnetic field-induced; they are absent without the latter. We point out also that the order of the phase transformation in the general case is connected with the orientation of the direction of the magnetic field with respect to the crystal axes and on the mutual ratios of the exchange constant, anisotropy, magnetic field, and elastic constant.

It turns out that (alternating) distortions of the ligands, which result in the inequivalence of the effective $g$ factors of neighboring spins in quasi- $1 D$ chains, can be supplemented in the real compounds by alternating distortions of the magnetic ions themselves (which leads to the spin-Peierls insta- bilities), as has been observed, e.g., in $\mathrm{CuGeO}_{3}$ [25]. In such a situation the two effects are additive in the formation of a spin gap for low-lying spin excitations. The values of the effects are naturally different. Although the effect studied in this work manifests itself only in some domain of values of the magnetic field, the spin-Peierls period doubling is not caused by the magnetic field. The critical temperature is determined from the condition of which of the instabilities becomes manifested first.

We suppose that the cooperative effects similar to the one studied in this paper have been observed in some rare-earth molybdates. There at low-temperature ESR studies that have observed two inequivalent magnetic centers, (see, e.g., Refs. 6-9), while for higher temperatures $[7,8]$ or higher magnetic fields (higher frequencies) [15] only one magnetic center was seen. The low-temperature specific heat exhibits features at temperatures higher than the transition to the magnetically ordered phase (which were hidden, though, due to the presence of a Schottky anomaly) [8]. Ultrasonic measurements have observed anomalies in the low-temperature dependences of the velocities of sound for acoustic [17] and optical branches [18] of the phonon spectra. Optical and magnetic investigations have observed a cooperative effect (in nonzero magnetic field), that was classified as being of the Jahn - Teller type $[6,16,26]$. Note that the transition to the magnetically ordered state was observed at much lower temperatures (see, e.g., Ref. 8, 18, 26), hence the cooperative effect we have discussed is observed in the paramagnetic (from the magnetic viewpoint) phase. It turns out that magnetic and elastic characteristics of some of those systems manifested the mentioned features only for nonzero external magnetic fields, with the order of the transition (which of the derivatives of the thermodynamic potential possesses singular behavior) being determined by the direction of the field, while for zero field for some compounds there were no observations of the Jahn - Teller-like cooperative effect [27].

Summarizing, we have studied a new realization of magnetoelastic interactions in low-dimensional magnetic systems. We have shown that low dimensional spin systems are unstable with respect to the spontaneous appearance of alternating distortions of the positions of the three-dimensional nonmagnetic atoms (ligands) that surround the magnetic ions. Those distortions are supplemented by the spontaneous onset of alternating effective $g$ factors of magnetic ions in the phase with short-range interactions. We have discussed the possibility of obser- 
ving the effect in a uniform external magnetic field, which in the situation considered produces both magnetization and staggered magnetization of the magnetic subsystem. We suppose that the effects, investigated theoretically in this work, have probably been observed in the low-temperature experiments in some quasi-low-dimensional magnetic compounds, in which the spin, charge, and orbital characteristics of the electron subsystem are substantially coupled to the elastic subsystem.

1. Colossal Magnetoresistance, Charge Ordering and Related Properties of Manganese Oxides, C. N. R. Rao and B. Raveau (eds.), World Scientific, Singapore (1998); Colossal Magnetoresistance Oxides, Y. Tokura (ed.), Gordon and Breach, Monographs in Cond. Matt. Science (1999); H. V. Löhneysen, J. Magn. Magn. Mater. 200, 532 (1999); M. Hase, I. Terasaki, and K. Uchinokura, Phys. Rev. Lett. 70, 3651 (1993); M. Isobe and Y. Ueda, J. Phys. Soc. Jpn. 65, 1178 (1996); Y. Maeno, H. Hashimoto, K. Yoshida, S. Nishizaki, T. Fujita, J. G. Bednorz, and F. Lichtenberg, Nature (London) 372, 532 (1994); T. Ishigiro, K. Yamaji, and G. Saito, Organic Superconductors, Springer-Verlag, Berlin (1998).

2. H. A. Jahn and E. Teller, Proc. Roy. Soc. A161, 220 (1937).

3. K. I. Kugel' and D. I. Khomskii, Usp. Fiz. Nauk 136, 621 (1982) [Sov. Phys. Usp. 25, 231 (1982)].

4. M. Date, H. Yamazaki, M. Motokawa, and S. Tazawa, Prog. Theor. Phys. Suppl. 46, 194 (1978); K. Oshima, K. Okuda, and M. Date, J. Phys. Soc. Jpn. 41, 475 (1976); ibid. 44, 757 (1978); K. Takeda, Y. Yoshino, K. Matsumoto, and T. Haseda, ibid. 49, 162 (1980).

5. A. Ochiai, T. Suzuki, and T. Kasuya, J. Phys. Soc. Jpn. 59, 4129 (1990); P. Bonville, A. Ochiai, T. Suzuki, and E. Vincent, J. Phys. (Paris) 4, 595 (1994); M. Kohgi, K. Iwasa, J.-M. Mignot, A. Ochiai, and T. Suzuki, Phys. Rev. B56, R11388 (1997); M. Kohgi, K. Iwasa, J.-M. Mignot, N. Pyka, A. Ochiai, H. Aoki, and T. Suzuki, Physica B230-232, 638 (1997); Physica B259-261, 269 (1999); M. Oshikawa, K. Ueda, H. Aoki, A. Ochiai, and M. Kohgi, J. Phys. Soc. Jpn. 68, 3181 (1999).

6. T. S. Stetsenko, A. M. Pshisukha, S. D. Elchaninova, and A. I. Zvyagin, Opt. Spectrosc. 34, 227 (1973); A. I. Zvyagin, T. S. Stetsenko, V. G. Yurko, and R. A. Vaishnoras, JETP Lett. 17, 135 (1973).

7. A. M. Pshisukha, A. S. Chernyi, and A. I. Zvyagin, Fiz. Nizk. Temp. 1, 473 (1975) [Sov. J. Low Temp. Phys. 1, 233 (1975)]; A. M. Pshisukha, A. I. Zvyagin, and A. S. Chernyi, ibid. 2, 339 (1976) [ibid. 2, 18 (1976)].

8. A. H. Cooke, M. M. Davidson, N. J. England, M. J. M. Leask, J. B. Lowry, A. C. Tropper, and M. R. Wells, J.
Phys. C9, L573 (1976); M. J. M. Leask, A. C. Tropper, and M. R. Wells, ibid. 14, 3481 (1981).

9. M. I. Kobets, V. A. Pashchenko, and E. N. Khatsko, Fiz. Nizk. Temp. 26, 370 (2000) [Low Temp. Phys. 26, 270 (2000)].

10. A. A. Zvyagin, Fiz. Nizk. Temp. 21, 825 (1995) [Low Temp. Phys. 21, 636 (1995)].

11. D. C. Dender, P. R. Hammar, D. H. Reich, C. Broholm, and G. Aeppli, Phys. Rev. Lett. 79, 1750 (1997).

12. M. Köppen, M. Lang, R. Helfrich, F. Steglich, P. Thalmeier, B. Schmidt, B. Wand, D. Pankert, H. Benner, H. Aoki, and A. Ochiai, Phys. Rev. Lett. 82, 4548 (1999); F. Steglich, P. Gegenwart, R. Helfrich, C. Langhammer, P. Hellmann, L. Donnevert, C. Geibel, M. Lang, G. Sparn, W. Assmus, G. R. Stewart, and A. Ochiai, Z. Phys. B103, 235 (1997); F. Steglich, M. Köppen, P. Gegenwart, T. Cichorek, B. Wand, M. Lang, P. Thalmeier, B. Schmidt, H. Aoki, and A. Ochiai, Acta Phys. Polon. A97, 1 (2000).

13. M. Oshikawa and I. Affleck, Phys. Rev. Lett. 79, 2883 (1997).

14. H. Shiba, K. Ueda, and O. Sakai, Preprint ISSP (1999).

15. A. G. Anders, S. V. Volotskii, and O. E. Zubkov, Fiz. Nizk. Temp. 19, 731 (1993) [Low Temp. Phys. 19, 526 (1993)].

16. D. Mihailović, J. F. Ryan, and M. C. K. Wiltshire, J. Phys. C20, 3047 (1987); D. Mihailovic and J. F. Ryan, ibid. 20, 3063 (1987).

17. I. M. Vitebskii, S. V. Zherlitsyn, A. I. Zvyagin, A. A. Stepanov, and V. D. Fil, Fiz. Nizk. Temp. 12, 1108 (1986) [Sov. J. Low Temp. Phys. 12, 626 (1986)].

18. G. A. Zvyagina and A. A. Zvyagin, Fiz. Nizk. Temp. 26, 482 (2000) [Low Temp. Phys. 26, 354 (2000)].

19. L. N. Bulaevskii, Zh. Eksp. Teor. Fiz. 43, 968 (1962) [Sov. Phys. JETP 16, 685 (1963)]; M. C. Cross, Phys. Rev. B20, 4606 (1979).

20. A. A. Belavin, A. M. Polyakov, and A. B. Zamolodchikov, Nucl. Phys. B241, 333 (1984).

21. See, e.g. V. E. Korepin, N. M. Bogoliubov, and A. G. Izergin, Quantum Inverse Scattering Method and Correlation Functions, Cambridge University Press (1993) and references therein.

22. M. C. Cross and D. S. Fisher, Phys. Rev. B19, 402 (1979).

23. See, e.g., R. J. Bursill, R. H. McKenzie, and C. J. Hamer, Phys. Rev. Lett. 83, 408 (1999).

24. V. M. Kontorovich and V. M. Tsukernik, Zh. Eksp. Teor. Fiz. 53, 1167 (1967) [Sov. Phys. JETP 26, 687 (1968)].

25. A. A. Gippius, E. N. Morozova, D. F. Khozeev, A. N. Vasil'ev, M. Baenitz, G. Dhalenne, and A. Revcolevschi, J. Phys. Condensed Matt. 9, L71 (2000).

26. V. I. Kut'ko, V. A. Pashchenko, and M. I. Kobets, Fiz. Nizk. Temp. 19, 1354 (1993) [Low Temp. Phys. 19, 962 (1993)].

27. V. I. Kut'ko, Yu. M. Kharchenko, A. A. Stepanov, and N. M. Nesterenko, Fiz. Nizk. Temp. 20, 361 (1994) [Low Temp. Phys. 20, 288 (1994)]. 